\title{
Chemoprevention of DMBA- Induced Mammary Tumors in Rats by a Combined Regimen of Alpha-Tocopherol, Selenium, and Ascorbic Acid
}

\author{
Margarida F. Dias, MD, Estela Sousa, MD, Silvério Cabrita, PhD, \\ João Patrício, $\mathrm{PhD}$, and Carlos F. Oliveira, $\mathrm{PhD}$ \\ Department of Experimental Pathology, Hospitais da Universidade de Coimbra, \\ Coimbra, Portugal
}

Abstract: This experimental study was designed to evaluate the efficacy of associated naturally occuring antioxidants in the prevention of chemically induced breast cancer using DMBA in virgin female Wistar rats. Rats were randomly allocated to three groups: control group (CG; $n=20)$, induction group (IG; $n=100$ ), and prevention group (PG; $n=70$ ). A single dose $(65 \mathrm{mg} / \mathrm{kg})$ of DMBA was administered in the IG and PG animals at 50 days of age. PG animals also received a single dose of alpha-tocopherol (200 mg/rat) 1 hour after DMBA administration and an association of selenium ( $\mathrm{p}-\mathrm{XSC}, 40 \mathrm{ppm} /$ day/rat) and ascorbic acid (540 mg/day/rat) in drinking water, daily, from carcinogenic induction until necropsy. Macroscopic study and pathology revealed a significantly lower development of neoplasms in the PG animals ( $p<0.05$ ); the number of rats with mammary tumors, breast cancer incidence, and the number of malignant breast tumors per rat as well as per tumor-bearing rat were significantly decreased in the PG animals. Other types of primary neoplasms existing in the IG animals totally disappeared in the PG animals. Immunostaining to hormone steroid receptors (ER and PR) and cathepsin D was similar in both groups. Overexpression of p53 and metal-

Address correspondence and reprint requests to: Margarida Figueiredo Dias, MD, Serviço de Ginecologia, Hospitais da Universidade de Coimbra, Apartado 9015, 3049 Coimbra Codex, Portugal, or e-mail: agoc@ mail.telepac.pt

(C) 2000 Blackwell Science Inc., 1075-122X/00/\$15.00/0

The Breast Journal, Volume 6, Number 1, 2000 14-19 lothioneine was significantly increased in the PG animals $(p<$ 0.05 ) and immunostaining to bromodeoxiuridin and Ki-67 was also stronger in the remaining tumors in the PG animals. These data thus add to the accumulating evidence that those micronutrients in combination seem to be effective in reducing the incidence of malignant tumors. Nevertheless, remaining tumors seem to present more aggressive behavior and characteristics of drug resistance.

Key Words: antioxidants, breast cancer, chemoprevention, micronutrients

$\mathrm{n}$ recent years the incidence of breast cancer has in-

creased almost everywhere. Alternatives to therapy need to be developed for breast cancer control. Toward this end, chemoprevention constitutes a valuable approach. It is mandatory to expand our efforts in identifying synthetic or naturally occuring agents that can inhibit the preneoplastic events preceding the occurence of clinically detectable cancers $(1,2)$. Several agents have been characterized as effective in cancer chemoprevention, but studies are not yet conclusive (3). Chemoprevention properties of vitamins and other micronutrients as single agents or complex mixtures (4-22), fatty acids and related substances (23-27), monoterpens (28), hormones, and related chemical agents $(1-3,29)$ have been 
studied in experimental models. To assess the efficacy of potential chemopreventive agents it is important to develop sensitive and selective methods for determining molecular, metabolic, and cellular transformations that are relevant to the process of carcinogenesis in mammary tissue.

Mammary carcinomas induced in rats by means of chemical carcinogens, mainly DMBA, seem to provide a good model to the understanding of mechanisms of susceptibility to carcinogens (30). Mammary tumors thus induced are hormone-dependent adenocarcinomas arising from terminal end buds (TEBs) on incompletely differentiated glands (31). These tumors bear a close resemblance to human breast cancer in their histologic and hormone-response patterns (29,32); nevertheless, immunostaining and molecular biomarkers have not been clearly defined in those experimental carcinomas (33-35).

In this study we investigated whether combined naturally occuring micronutrients with antioxidant molecular activity inhibit the incidence of DMBA-induced mammary tumors in Wistar rats. We also describe the characteristics of aggressiveness and the drug resistance of remaining tumors using immunostaining for breast cancer biochemical markers.

\section{MATERIALS AND METHODS}

One hundred ninety outbred virgin female Wistar rats were obtained from the Instituto Gulbenkian de Ciência (Lisboa, Portugal). Animals were housed four in each cage in a temperature- $\left(23 \pm 2{ }^{\circ} \mathrm{C}\right)$ and humidity$(50-55 \%)$ controlled facility on a 12-hour light, 12hour dark cycle and fed a standard laboratory chow. Food and water were available ad libitum and mean water consumption was calculated every week.

At 50 days of age, rats were randomly allocated to three groups: control group (CG; $n=20)$, induction group (IG; $n=100$ ), and prevention group (PG; $n=$ $70)$. Biopsies of the right cervical mammary gland were performed in all animals under general anesthesia (ketamine intramuscularly, $5 \mathrm{mg} / 100 \mathrm{~g}$ ). CG animals received no more drugs until the end of the experience. IG and PG rats were given a single dose $(65 \mathrm{mg} / \mathrm{kg})$ of $7,12-$ dimethylbenz[a]anthracene (DMBA) in olive oil via an intragastric tube. PG rats also received a single dose of alpha-tocopherol (200 mg/rat) 1 hour after DMBA administration via an intragastric tube and a solution of selenium (p-XSC, $40 \mathrm{ppm} / \mathrm{day} / \mathrm{rat}$ ) and ascorbic acid $(540 \mathrm{mg} /$ day/rat$)$ in drinking water, daily, from carcinogenic induction until necropsy.
All animals were kept under previously described environmental conditions and they were palpated weekly to determine the appearance, size, and location of tumors. They were weighed monthly.

Twenty-four weeks after carcinogenic induction rats were killed by cervical dislocation 1 hour after intravenous bromodeoxiuridin (BrdUr) administration (50 $\mathrm{mg} / \mathrm{kg}$ ). The rats that died before 24 weeks were excluded from this study.

Macroscopic tumors were excised and the size, measured in two perpendicular dimensions, was estimated from the mean diameter. Mammary glands free of nonpalpable tumors were also removed and biopsies of the following organs were performed: trachea, heart, lungs, esophagus, liver, spleen, pancreas, bowel, bladder, and internal genital organs.

\section{Histologic Evaluation}

Tissue fragments were fixed in $10 \%$ formalin and a $5 \mu \mathrm{m}$ section was obtained from the paraffin block and stained with hematoxylin and eosin for histologic examination. Breast disease pathology and histologic type were evaluated by application of the same pathologic criteria used for the classification of human tumors (36). Breast cancer grading was performed according to the Nottingham modification of the Bloom and Richardson system.

\section{Assessment of Staining}

Estrogen receptors (ERs, clone 1D5, Dako) and progesterone receptors (PRs, A009850, Dako) were immunostained on frozen sections. Tumors with cytoplasmic ER and PR greater than $5 \mathrm{fmol} / \mathrm{mg}$ of protein were considered positive.

Immunohistochemistry was performed for p53 (M700101, Dako), pS2 (A009501, Dako), c-erbB-2 (A0485, Dako), cathepsin D (A056101, Dako), metallothioneine (M063901, Dako), Ki-67 (A004701, Dako), and bromodeoxiuridin (BrdUr, clone BU20a, Boehringer Mannheim). Immunohistochemical analyses were performed according to previously published methods (37).

\section{Statistical Analysis}

Differences in tumor incidence and immunostaining between groups were determined using the Fisher's test. Differences in rat body weight and tumor size were statistically evaluated by one-way analysis of variance (ANOVA) followed by chi-square tests. Values of $\mathrm{p}<$ 0.05 were considered significant. 


\section{RESULTS}

Mammary gland biopsies performed at 50 days of age exhibited similar characteristics of mammary developmental stage: terminal end buds (TEBs) composed of three to six layers of medium-size epithelial cells with a fairly constant mitotic activity as predominantly mammary structures. All rats presented the same mammary gland developmental stage by the time of carcinogenic induction.

Total body weight did not change significantly among the groups of rats throughout the experimental period. The PG animals' body weight 24 weeks after DMBA administration was less than that for the CG and IG animals, but the difference was not significant. No statistically significant difference was observed when comparing the body weight of rats with breast cancer to that of healthy IG and PG rats.

CG rats were all healthy and alive at the end of the experience. Ten rats in each of the other groups died before necropsy and were excluded from the study. IG rats displayed $35(39 \%)$ benign breast disorders and 56 $(62 \%)$ breast carcinomas. Macroscopic study and histopathologic examination of IG animals showed 7 primary neoplasms and 14 benign lesions of other tissues and organs, mainly benign ovarian disorders. PG rats presented no other tumors but breast tumors: 17 (28\%) benign breast disorders and $9(15 \%)$ breast carcinomas (Table 1).

Statistical analysis comparing breast disorders demonstrates that rat body weight and the average diameter of macroscopic breast tumors did not differ significantly in IG and PG rats. Nevertheless, the total number of rats with malignant tumors, breast cancer incidence, and the total number of malignant breast carcinomas were significantly decreased in PG. Moreover, the number of malignant breast tumors per rat as well as per tumor-

Table 1. Benign Disorders and Malignant Tumors in IG and PG Rats

\begin{tabular}{lccccc}
\hline & \multicolumn{2}{c}{ IG $(n=90)$} & & \multicolumn{2}{c}{ PG $(n=60)$} \\
\cline { 2 - 3 } Number of lesions & BEN & MAL & & BEN & MAL \\
\hline Breast & 35 & 56 & & 17 & 9 \\
Ovary & 6 & - & & - & - \\
Salivary gland & 4 & - & & - & - \\
ORL & 1 & 3 & & - & - \\
Lymphoma & - & 2 & & - & - \\
Lungs & - & 1 & & - & - \\
Liver & 2 & - & & - & - \\
Pancreas & - & 1 & & - & - \\
Bowel & 1 & - & & - \\
\end{tabular}

bearing rat were significantly decreased in animals receiving alpha-tocopherol, selenium, and ascorbic acid (Table 2).

PG rats never presented preinvasive ductal carcinoma in situ or atypical hyperplasias. Benign breast disorders were composed of proliferative lesions without atypia in PG animals (Table 3). No significant difference was reported in histologic grade and microscopic pathology. No breast cancer presented vascular invasion and no metastatic lesions were found in all biopsies performed (Table 4). ER (43\% IG and 33\% PG) and PR (18\% IG and $22 \% \mathrm{PG}$ ) contents of the tumors were not significantly different (Table 5).

Concerning immunohistochemical staining, 29\% breast cancer IG rats presented positivity for p53 overexpression; nevertheless, $78 \%$ of breast carcinomas in PG rats displayed $\mathrm{p} 53$ accumulation, and this difference was statistically significant. All breast carcinomas were c-erbB-2 negative and only one IG breast cancer was considered pS2 positive. Immunostaining for cathepsin D was higher in IG than in PG rats, although the difference wasn't significant.

Metallothioneine overexpression was found in 67\% of PG breast cancers and occasionally $(10 \%)$ in IG breast carcinomas; MT-positive tumor cells were significantly increased in PG rats. Finally, considering immunohistochemical Ki-67 and BrdUr expression, staining intensity was significantly stronger in PG breast carcinomas.

\section{DISCUSSION}

We have to enhance our understanding of the role of naturally occuring antioxidants in cancer prevention and their possible use in intervention trials for the prevention of cancer in humans. Selenium alone seems to inhibit both the initiation and postinitiation phases of carcinogenesis in animal models (21), and in vitro studies have been performed to investigate the antiproliferative action of selenium (38). The mechanisms and forms

Table 2. Pathology of Breast Tumors in IG and PG Rats

\begin{tabular}{|c|c|c|c|c|}
\hline & \multicolumn{2}{|c|}{$N=90$} & \multicolumn{2}{|c|}{$N=60$} \\
\hline & $n$ & $(\%)$ & $n$ & $(\%)$ \\
\hline Malignant breast tumors & \multicolumn{2}{|c|}{ IG $(n=56)$} & \multicolumn{2}{|c|}{ PG $(n=9)$} \\
\hline DCIS & 3 & $(5.4)$ & - & \\
\hline Invasive ductal carcinoma & 53 & $(94.6)$ & 9 & $(100)$ \\
\hline Benign breast disorders & \multicolumn{2}{|c|}{ IG $(n=35)$} & \multicolumn{2}{|c|}{ PG $(n=9)$} \\
\hline Glandular hyperplasia & 24 & $(68.6)$ & 13 & $(76.5)$ \\
\hline Atypical hyperplasia & 7 & $(20)$ & - & \\
\hline Fibroadenoma & 3 & $(8.6)$ & 4 & $(23.5)$ \\
\hline Papillary lesions & 1 & $(2.8)$ & - & \\
\hline
\end{tabular}


Table 3. Pathologic Features and Aggressiveness of Malignant Breast Tumors in IG and PG Rats

\begin{tabular}{|c|c|c|c|}
\hline & $N=90$ & $N=60$ & \\
\hline & IG $(n=53)$ & PG $(n=9)$ & Fisher $(p<0.05)$ \\
\hline GI & $49(93 \%)$ & $9(100 \%)$ & NS (0.4839) \\
\hline GII & $4(7 \%)$ & - & \\
\hline GIII & - & - & \\
\hline Invasive ductal carcinoma & & & \\
\hline $\begin{array}{l}\text { (no other specification) } \\
\text { Invasive tubular carcinoma }\end{array}$ & $\begin{array}{c}50(94 \%) \\
2(4 \%)\end{array}$ & $\begin{array}{c}9(100 \%) \\
-\end{array}$ & NS (0.5485) \\
\hline Invasive papillary carcinoma & $1(2 \%)$ & - & \\
\hline Lymphoplasmacytic infiltrate & $13(25 \%)$ & $3(33 \%)$ & NS (0.1637) \\
\hline Fibrosis & $29(55 \%)$ & $6(67 \%)$ & NS (0.1426) \\
\hline Necrosis & $2(4 \%)$ & - & \\
\hline Vascular invasion & - & - & \\
\hline Metastatic lesions & - & - & \\
\hline
\end{tabular}

of selenium that are responsible for chemoprevention in laboratory animals need to be elucidated $(15,20,21)$.

Previous articles reported the anticarcinogenic activity of aliphatic selenocyanates, suggesting that the anticarcinogenic potency of those compounds is correlated with their ability to inhibit DMBA-DNA adduct formation in mammary cells $(10,22)$. Other studies revealed that selenium supplementation clearly inhibits DMBAinduced mammary tumorigenesis in rats and mice (511). Epidemiologic reports show a higher cancer incidence in geographic areas of low selenium intake (39).

Combined chemopreventive effects of alpha-tocopherol and selenium in rodent tumors seem to be more promising than that of selenium or alpha-tocopherol alone $(3,13,14,18,19)$. On the other hand, the effect of ascorbic acid alone in some tumor model systems is not consistent or appears to be weak (17). The combined effect of selenium and vitamin C probably depends on the chemical form of the selenium compound (12).

This experimental study was designed to evaluate the combined effect of the referred three micronutrients. Results point to an effective chemopreventive action of this mixture in reducing the incidence and preventing the development of malignant tumors in this animal model.

Table 4. Estrogen Receptor (ER) and Progesterone Receptor (PR) Contents of Malignant Breast Tumors

\begin{tabular}{cccc}
\hline & IG & PG & Fisher $(p<0.05)$ \\
\hline ER+ & & & \\
$n$ & $24 / 56$ & $3 / 9$ & NS $(0.0817)$ \\
$\%$ & 43 & 33 & \\
PR+ & & & NS $(0.3938)$ \\
$n$ & $10 / 56$ & $2 / 9$ & \\
$\%$ & 18 & 22 & \\
\hline
\end{tabular}

After carcinogenic induction, only a few mammary tumors developed and no other primary neoplasms were observed in animals receiving selenium, alpha-tocopherol, and ascorbic acid. Breast cancer incidence, total number of malignant tumors, and the number of breast carcinomas per rat as well as per tumor-bearing rat were significantly decreased in PG animals. So the use of selenium, alpha-tocopherol, and ascorbic acid in a combined regimen seems to be useful as a suppressive chemopreventive regimen for breast cancer and other neoplasms in this particular animal model. Daily intake of those micronutrients beginning immediately after carcinogenic induction causes a significantly lower development of malignancies.

Nevertheless, these naturally occuring antioxidants displayed no inhibitory effect on tumor growth in established tumors. Finally, this remaining group of tumors seems to be associated with a more aggressive behavior and with resistance against anticancer drugs, since they exhibited higher levels of p53 and metallothioneine. Mammary tumors induced in the presence of those antioxidants possess characteristics distinct from the tumors

Table 5. Molecular Tumor Markers: Results of Immunohistochemistry in Malignant Tumors

\begin{tabular}{|c|c|c|c|}
\hline & IG $(N=56)$ & $\mathrm{PG}(N=9)$ & \\
\hline & $n(\%)$ & $n(\%)$ & Fisher $(p<0.05)$ \\
\hline $\mathrm{p} 53^{+}$ & $16(29)$ & $7(78)$ & 0.0001 \\
\hline $\mathrm{pS} 2^{+}$ & $1(2)$ & - & \\
\hline c-erbB-2 $2^{+}$ & - & - & \\
\hline Cathepsin $\mathrm{D}^{+}$ & $31(55)$ & $4(44)$ & NS (0.0973) \\
\hline Metallothioneine $^{+}$ & $5(10)$ & $6(67)$ & 0.0001 \\
\hline $\mathrm{Ki} 67>50 \%$ & $10(18)$ & $4(44)$ & 0.001 \\
\hline BrdUr $>50 \%$ & $16(29)$ & $4(44)$ & 0.0237 \\
\hline
\end{tabular}


occuring in their absence. Moreover, the appearance of new tumors during micronutrient intake suggests that there is a different class of tumors not susceptible to prevention in this way.

Selection of chemopreventive agents need not to be limited to naturally occuring compounds, and combined effects of synthetic and/or naturally occuring agents with different modes of action should be examined (1) and selected on the basis of efficacy versus toxicity considering the need of long-term intake.

Finally, the availability of increasingly sophisticated biochemical tools in molecular epidemiology in recent years provides a better understanding which undoubtedly offers opportunities for defining and modulating breast cancer risk by means of agents that alter critical steps in the multistage carcinogenic process.

\section{Acknowledgment}

This study was supported by a grant of the "Comissão de Fomento da Investigação em Cuidados de Saúde" from the Ministry of Health of Portugal.

\section{REFERENCES}

1. Wattenberg LW. Chemoprevention of cancer by naturally occurring and synthetic compounds. In: Wattenberg L, Lipkin M, Boone CW, Kelloff GJ, eds. Cancer chemoprevention. Boca Raton, FL: CRC Press, 1992:19-39.

2. Greenwald P, Malone WF, Cerny ME, Stern HR. Cancer prevention research trials. Adv Cancer Res 1993;61:1-23.

3. El-Bayoumy K. Evaluation of chemopreventive agents against breast cancer and proposed strategies for future clinical intervention trials. Carcinogenesis 1994;15:2395-429.

4. Ip C, Donald JL, Henry JT. Selenium-enriched garlic inhibits the early stage but not the late stage of mammary carcinogenesis. Carcinogenesis 1996;17:1979-82.

5. Ip C, Ip MN. Chemoprevention of mammary tumorigenesis by a combined regimen of selenium and vitamin A. Carcinogenesis 1981;2:915-18.

6. Medina D, Shepherd F. Selenium-mediated inhibition of mouse mammary tumorigenesis. Cancer Lett 1980;8:241-45.

7. Ip C. Prophylaxis of mammary neoplasia by selenium supplementation in the initiation and promotion phase of chemical carcinogenesis. Cancer Res 1981;41:4386-90.

8. Medina D, Shepherd F. Selenium-mediated inhibition of 7,12-dimethylbenz[a]anthracene-induced mouse mammary tumorigenesis. Carcinogenesis 1981;2:451-55.

9. Ip C, Ganther HE. Activity of methylated forms of selenium in cancer prevention. Cancer Res 1990;50:1206-11.

10. Liu J, Gilbert K, Parker HM, Haschek NM, Milner JA. Inhibition of 7,12-dimethylbenz[a]anthracene-induced mammary tumors and DNA adducts by dietary selenite. Cancer Res 1991;51:4613-17.
11. Ip C, El-Bayoumy K, Upadhyaya P, Ganther H, Vadhanavikit $\mathrm{S}$, Thompson $\mathrm{H}$. Comparative effect of inorganic and organic selenocyanate derivatives in mammary cancer chemoprevention. Carcinogenesis 1994;15:187-92.

12. Novotny JA, Milner JA. Impact of ascorbic acid on selenium-induced growth inhibition of canine mammary tumor cells in vitro. J Nutr Biochem 1993;4:341.

13. Hovarth P, Ip C. Synergistic effect of vitamin E and selenium in the chemoprevention of mammary carcinogenesis in rats. Cancer Res 1983;43:5335-41.

14. Takada H, Hirooka T, Hatano T, Hamada Y, Yamamoto M. Inhibition of 7,12-dimethylbenz[a]anthracene-induced lipid peroxidation and mammary tumor development in rats by vitamin E in conjunction with selenium. Nutr Cancer 1992;17:115-22.

15. Thompson HJ, Wilson A, Lu J, et al. Comparison of the effects of an organic and an inorganic form of selenium on a mammary carcinoma cell line. Carcinogenesis 1994;15:183-86.

16. Rao AN, Rao AR, Jannu LN, Hussain SP. Chemoprevention of 7,12-dimethylbenz[a]anthracene-induced mammary carcinogenesis in rat by the combined actions of selenium, magnesium, ascorbic acid and retinyl acetate. Jpn J Cancer Res 1990;81:1239-46.

17. Abul-Hajj YJ, Kelliher M. Failure of ascorbic acid to inhibit growth of transplantable and dimethylbenz[a]anthraceneinduced rat mammary tumors. Cancer Lett 1982;17:67-73.

18. Harman D. Dimethylbenz[a] anthracene induced cancer: inhibitory effect of dietary vitamin E. Clin Res 1969;17:125.

19. Ip C. Dietary vitamin E intake and mammary carcinogenesis in rats. Carcinogenesis 1982;3:1453-56.

20. Ip C, Lisk DJ. Characterization of tissue selenium profiles and anticarcinogenic responses in rats fed natural sources of selenium-rich products. Carcinogenesis 1994;15:573-76.

21. Ip C, Lisk DJ. Efficacy of cancer prevention by highselenium garlic is primarily dependent on the action of selenium. Carcinogenesis 1995;16:2649-52.

22. Ip C, Vadhanavikit S, Ganther H. Cancer chemoprevention by aliphatic selenocyanates: effect of chain length on inhibition of mammary tumors and DMBA adducts. Carcinogenesis 1995;16:35-38.

23. El-Sohemy A, Bruce WR, Archer MC. Inhibition of rat mammary tumorigenesis by dietary cholesterol. Carcinogenesis 1996;17:159-62.

24. Nakayama M, Ju HR, Sugano M, et al. Effect of dietary fat and cholesterol on dimethylbenz[a]anthracene-induced mammary tumorigenesis in Sprague-Dawley rats. Anticancer Res 1993;13:691-98.

25. Welsch CW. Relationship between dietary fat and experimental mammary tumorigenesis: a review and critique. Cancer Res 1992;52:2040-48.

26. Melhem MF, Gabriel HF, Eskander ED, Rao KN. Cholestyramine promotes 7,12-dimethylbenz[a]anthracene induced mammary cancer in Wistar rats. Br J Cancer 1987;56: $45-48$.

27. Noguchi M, Minami M, Yagasaki R, et al. Chemopre- 
vention of DMBA-induced mammary carcinogenesis in rats by low-dose EPA and DHA. Br J Cancer 1997;75:348-53.

28. Elegbede JA, Elson CE, Qureshi A, Tanner MA, Gould MN. Inhibition of DMBA-induced mammary cancer by the monoterpene d-limonene. Carcinogenesis 1984;5:661-64.

29. Welsch CW. Host factors affecting the growth of carcinogen-induced rat mammary carcinomas: a review and tribute to Charles Brenton Huggins. Cancer Res 1985;45:3415-43.

30. Russo J, Wilgus G, Russo IH. Susceptibility of the mammary gland to carcinogenesis. Am J Pathol 1979;93:721-33.

31. Russo IH, Russo J. Developmental stage of the rat mammary gland as determinant of its susceptibility to 7,12 dimethylbenz[a]anthracene. Cancer Inst 1978;61:1439-45.

32. Russo J, Gusterson BA, Rogers AE, Russo IH, Wellings R, van Zwieten MJ. Biology of disease: comparative study of human and rat mammary tumorigenesis. Lab Invest 1990;62:244-73.

33. Murad T, von Haam E. Studies of mammary carcinoma induced by 7,12-dimethylbenz[a] anthracene administration. Cancer Res 1972;32:1404-15.
34. Hilf R, Goldenberg H, Gruenstein M. Lack of correlation between morphological and biochemical parameters in mammary adenocarcinomas of rats induced with 7,12-dimethylbenz[a]anthracene. Cancer Res 1970;30:1223-30.

35. Russo J, Saby J, Isenberg WM, Russo IH. Pathogenesis of mammary carcinomas induced in rats by 7,12-dimethylbenz[a]anthracene. J Natl Cancer Inst 1977;59:435-45.

36. World Health Organization. Histological typing of breast tumours. In: International histological classification of tumours, 2nd ed. Geneva: World Health Organization, 1981:19.

37. Bratthauer GL. The avidin-biotin complex (ABC) method. Meth Mol Biol 1994;34:175-84.

38. Wu L, McGarry L, Lanfear J, Harrison PR. Altered selenium-binding protein levels associated with selenium resistance. Carcinogenesis 1995;16:2819-24.

39. Garland M, Morris JS, Stampfer MJ. Prospective study of toenail selenium levels and cancer among women. J Natl Cancer Inst 1995;87:497-505. 